C-A/AP/\#193

March 2005

\title{
Possible NSRL Beams Compatible with Gold-Gold Collisions in RHIC
}

P. Thieberger

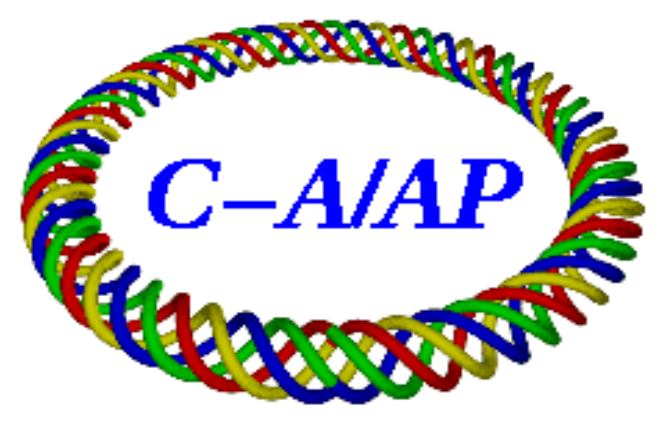

Collider-Accelerator Department Brookhaven National Laboratory

Upton, NY 11973 


\section{Possible NSRL beams compatible with gold-gold collisions in RHIC}

\section{P. Thieberger}

First we will try to make a list of beams that may be compatible from the point of view of rigidities and charge states, assuming that we can have a "flipping" stripper installed in section 28 and an object stripper in its present location following MP7, or one in the equivalent position following MP6.

Figure 1 shows the different stripper combinations that have been considered.

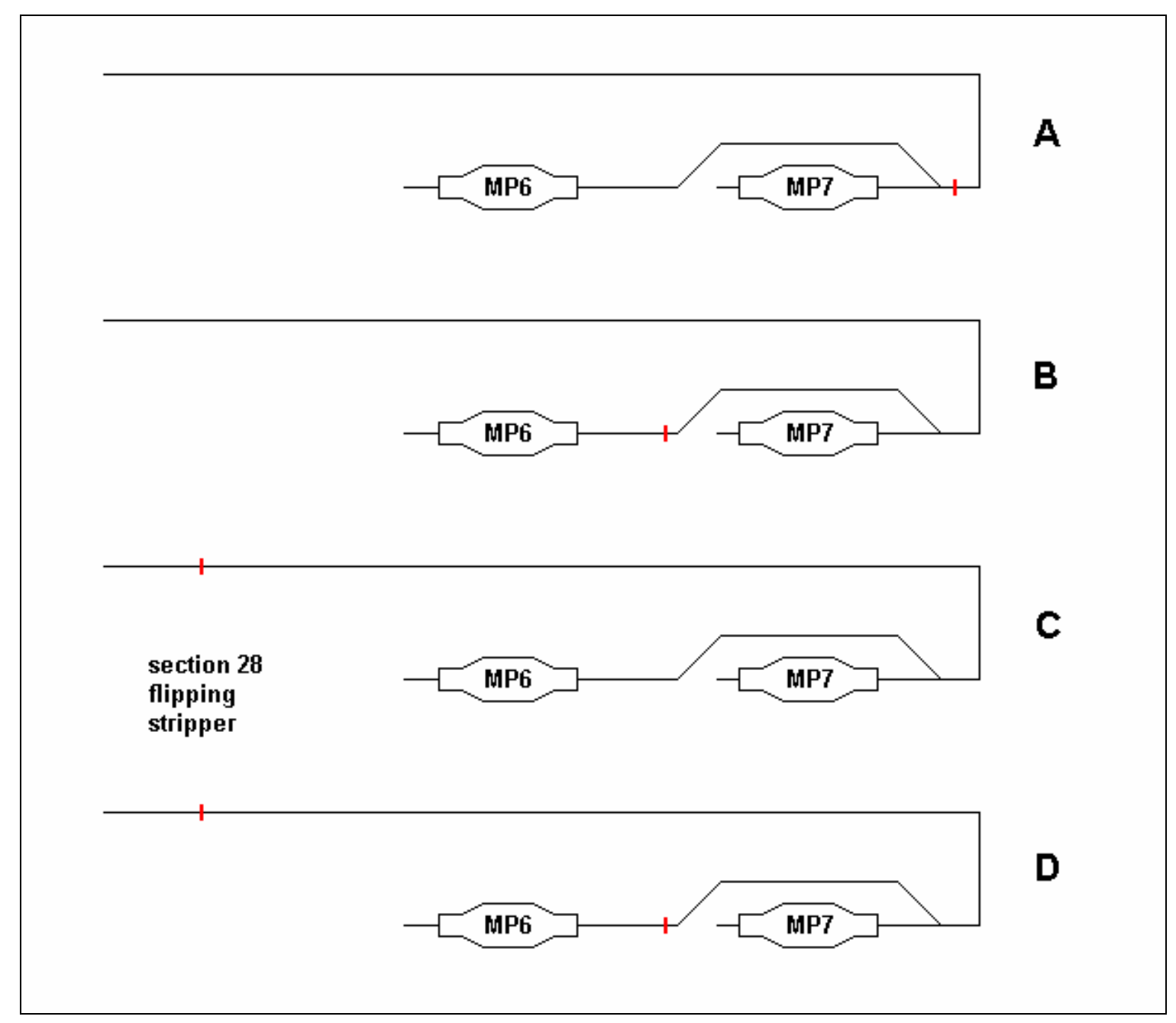

Fig. 1 Schematic representation of the stripper locations and possible combinations that have been considered in this report. It is assumed that the stripper in section 28 can be cycled between a foil and a blank in about one second, and that the other strippers are not cycled. Configuration $\mathrm{A}$ is the normal configuration used for gold beams whether they come form MP7 or from MP6.

In all cases, the magnetic rigidity in TTB up to section 28 must be adjusted to be the same than for the $182 \mathrm{MeV}$ gold beam used for RHIC, since the large dipole magnets in the line cannot be switched rapidly. In section 28 and beyond, there are only quadrupole lenses that have been shown to be adjustable in about 1 second. Table 1 lists the cases we have considered. 
Table 1. The listed beams would be compatible with the standard 182 gold beam used by RHIC. The "configuration" column refers to the labels shown in fig. 1. Q1 is the charge state used coming out of the accelerator and Q2 (if different from Q1) is generated in one of the external strippers. In the last three cases (configuration $\mathrm{C}$ ) the second gold stripping takes place in the section-28 stripper that in these cases can be fixed, but there are potential problems with these cases as with other possible beams (see text).

\begin{tabular}{||l||c|c|c|c|c|c|}
\hline \hline Beam & Machine & Configuration & Energy & $\begin{array}{l}\text { Terminal } \\
\text { voltage }\end{array}$ & Q1 & Q2 \\
\hline & & & $(\mathrm{MeV})$ & $(\mathrm{MV})$ & & \\
\hline \hline H 2 & MP6 or 7 & A & 17.5 & 8.7 & 1 & 1 \\
\hline C 12 & MP7 & B & 73.1 & 12.2 & 5 & 5 \\
\hline O 16 & MP7 & B & 107.4 & 13.4 & 7 & 7 \\
\hline Si 28 & MP7 & D & 80.0 & 8.8 & 8 & 13 \\
\hline Ti 48 & MP6 or 7 & $\mathrm{C}$ & 46.5 & 11.6 & 3 & 15 \\
\hline Ni 58 & MP6 or 7 & $\mathrm{C}$ & 68.5 & 13.7 & 4 & 18 \\
\hline Co59 & MP6 or 7 & $\mathrm{C}$ & 67.5 & 13.5 & 4 & 18 \\
\hline \hline
\end{tabular}

We will now briefly discuss various aspects of these possibilities.

\section{Deuterium}

Whether the deuterium comes from form MP6 or from MP7, this is the easiest and most straightforward case. The standard object stripper is used, and even though it isn't needed for the deuterium, it will not affect this beam in a very significant way. Slit regulation will have to be rapidly switched as one or the other beam is delivered, but this is always the case when the two injectors are used in rapid sequence. This beam has been delivered to NSRL before, but in that case the delivery was interrupted during RHIC gold fills. It should be possible to deliver this beam to NSRL between AGS cycles, thus not requiring interruptions. The slight retuning of section \#12 that was apparently necessary during that past run should not be required. It would take some further testing to find a way to avoid this retuning.

\section{Carbon and Oxygen}

Theses are rather straightforward cases too. They only require moving the standard oscillating stripper for gold to MP6. Configuration " $\mathrm{B}$ " is proposed, but configuration " $\mathrm{D}$ " could also be used if one wanted to fully strip these beams to obtain maximum energy in the Booster. The disadvantage of doing this is that the standard use of wire-scatterers to adjust emittance and intensity would not work. 


\section{Silicon}

This is the first case that requires the flipping stripper in section 28, unless the lower Booster energy corresponding to charge state 8 is acceptable. In that case configuration " $\mathrm{B}$ " could be used instead of "D".

\section{Titanium Nickel and Cobalt}

These three cases would be implemented with gas stripping in the Tandem and using stripper configuration "C". Also in all these cases the Booster output energy will be somewhat lower than can be achieved in the absence of the rigidity constraint. The maximum bending power of the dipoles in TTB is more than adequate to transport the charge 12 gold, but some power supply modifications would be required to implement this configuration.

The most serious potential problem with these beams is that the gold performance for RHIC may be affected. There are three ways this may happen. First, the gold emittance growth in the stripper foil if located in section 28 will be larger than if that foil is in its standard location where the beam is smaller. Second, as the thickness of the stripper foil changes due to effects caused by the beam, the energy loss changes, slightly affecting the beam energy delivered to the Booster. Finally, the possibility that defocused fractions of the gold beam, corresponding to charge states not being used, may adversely affect the Booster inflector is also a concern ${ }^{1)}$. On the other hand, there may be an advantage for RHIC in making that foil longer lasting and more reliable, as the beam spot is larger. Further studies and tests would be required to evaluate potential impacts on RHIC operations.

There are possible countermeasures to mitigate these potential difficulties. A low- $\beta$ insertion could make the beam spot small, feedback from the Booster could in principle help correct the energy drift, and a modest chicane would get rid of the unwanted charge-states

\section{Conclusions}

When RHIC is running gold-gold collisions it should be relatively straightforward to deliver uninterrupted deuterium, oxygen or silicon beams to NSRL. The flipping stripper in TTB section \#28 would be needed for silicon. Titanium, cobalt or nickel could work too, but several issues would need to be investigated, any one of which could make the implementation of these options more expensive. On the other hand, the savings from compatible RHIC - NSRL operations would be considerable.

In a separate report we shall address NSRL beam compatibilities with beams in RHIC other than gold. For most of these cases the flipping stripper would also be required.

\section{Acknowledgements}

Enlightening discussions with Jim Alessi, John Benjamin, Chuck Carlson, Dannie Steski and Woody Glenn are gratefully acknowledged.

1) C. J. Gardner, private communication. 